\title{
Health-Related Quality of Life in Stroke Survivors at the University Hospital of the West Indies
}

\author{
Jodian A. Pinkney, b, d, e, Francene Gayle ${ }^{\mathrm{a}, \text { b, d }}$, Kathryn Mitchell-Fearon ${ }^{\mathrm{c}, \mathrm{d}}$, \\ Jasneth Mullings ${ }^{b, d}$
}

\begin{abstract}
Background: Stroke remains a major contributor to mortality and morbidity both locally and globally. To date, there has been no study examining the impact of stroke on quality of life (QOL) in the Jamaican population. Our study was the first to look at QOL among Jamaican stroke survivors across the vast spectrum of stroke severity ranging from mild to severe. We also aimed to identify variables that led to decreased QOL in this cohort when compared to healthy adults.

Methods: This was a hospital-based case-control study comparing the QOL of 50 stroke patients admitted to the University Hospital of the West Indies (UHWI) in Jamaica between 2012 and 2013 to that of 50 apparently healthy adults (AHAs). Stroke severity was calculated using the National Institutes of Health stroke scale (NIHSS) at admission. The health-related quality of life in stroke patients (HRQOLISP) tool was used to attain the QOL score in each group.
\end{abstract}

Results: Of the 108 patients that were coded as having an acute ischemic stroke in the year 2012, $38(35.1 \%)$ were deceased and 20 $(18.5 \%)$ were lost to follow-up at the time of the study. There was no statistical difference between the stroke group and the AHAs with regard to age, race and traditional risk factors for stroke. Within the stroke population, the mean age was $61 \pm 17.8$ years. The mean age among the AHAs was $60 \pm 13.1$ years. Of the stroke survivors, 25 $(50 \%)$ were male and $25(50 \%)$ were female. Forty-five $(90 \%)$ patients were hypertensive. Health-related quality of life (HRQOL) was significantly reduced across most domains when compared to AHAs $(\mathrm{P}=0.0001)$. Greater stroke severity, presence of depression and previous stroke were all significantly associated with worse QOL.

Manuscript accepted for publication April 07, 2017

aDepartment of Medicine, University of the West Indies, Mona Campus, Mona Jamaica, West Indies

${ }^{b}$ Faculty of Medical Sciences, University of the West Indies, Mona Campus, Mona, Jamaica, West Indies

'Department of Community Health and Psychiatry, University of the West Indies, Mona, Jamaica, West Indies

${ }^{\mathrm{d}}$ These authors contributed equally to this work.

${ }^{e}$ Corresponding Author: Jodian A. Pinkney, Department of Medicine, University of the West Indies, Mona Campus, Mona Jamaica, West Indies. Email: jodian.pinkney@gmail.com

doi: https://doi.org/10.14740/jnr422w
Conclusions: In Jamaica, HRQOL among stroke survivors at the UHWI is consistently and significantly lower than that of healthy adults. Strategic interventions that target stroke severity, depression and non-adherence to secondary prevention regimens must be implemented in order to improve patient outcomes.

Keywords: Quality of life; Stroke; Stroke survivors; Jamaica; HRQOL

\section{Introduction}

According to the Jamaica health and lifestyle survey done in 2007 , the prevalence of stroke in Jamaica is approximately $1.4 \%$ [1]. In the year 2000, stroke was found to be the leading cause of death in Jamaica [2]. Globally stroke is thought to be the second leading cause of death accounting for a quarter of deaths worldwide [3]. Despite these daunting figures, mortality-based rates used in isolation remain inadequate to determine disease burden, health outcomes and intervention outcomes of a disease, all of which are important for planning national and institutional policies. In the US, it is estimated that $85 \%$ of people who experience a stroke survive [4]. Additionally, $8 \%$ of these survivors go on to have another stroke within 1 year with increasing risk for each additional year of life [5]. The impact of stroke on the life of its victim must therefore be quantified and examined.

Health-related quality of life (HRQOL) is being increasingly recognized as a better marker for disease burden within a population. The World Health Organization (WHO) defines quality of life (QOL) as "individuals' perception of their position in life in the context of the culture and value systems in which they live and in relation to their goals, expectations, standards and concerns" [6]. In the past, QOL of a disease was expressed using the common metric quality of life years (QALYs). QALYs combine information pertaining to length of time alive and QOL measured typically from 0 (death) to 1 (perfect health). For persons that had suffered a major stroke, previous studies had elicited QALY scores ranging between -0.02 and $0.71[4,7]$. How is this interpreted? Essentially if a major stroke has a QOL of 0.4 , then every year spent in that state of health is viewed as 0.4 years by that individual, i.e. if that person lives for 10 years, they would have preferred to live 


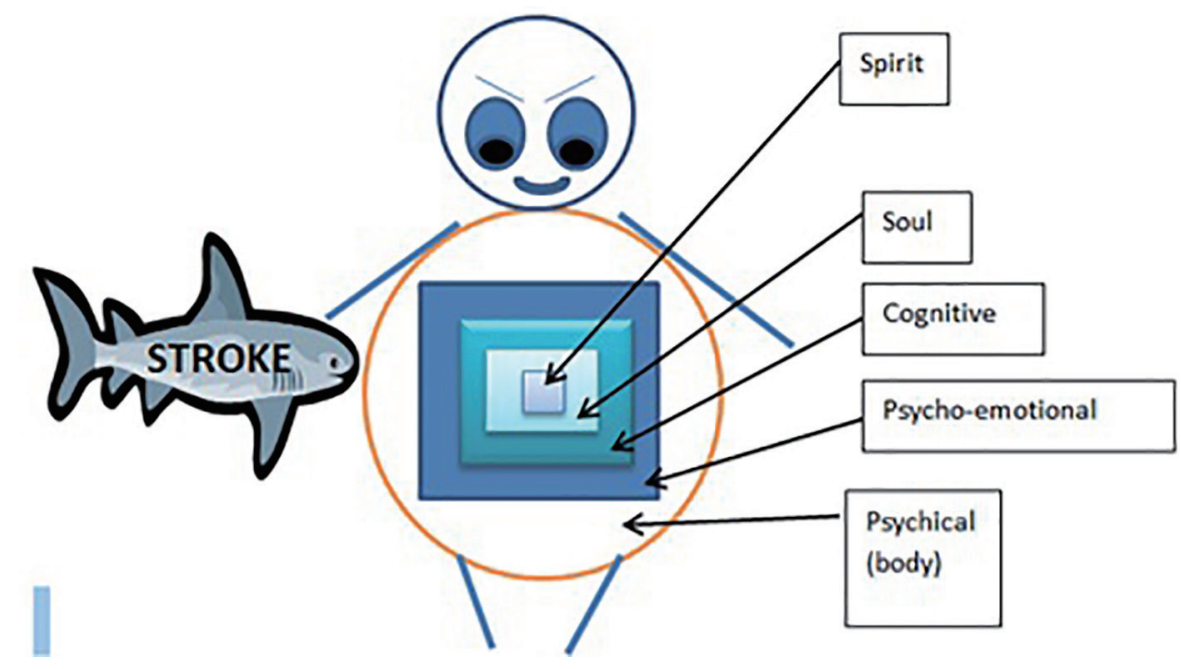

Figure 1. Seed of life model (SOLM). The seed of life model shows that the human is like a matryoshka doll. The spiritual sphere comprising of the spirit and the soul domains of quality of life represent the inner most protected core dolls. The physical sphere comprising of the physical, psycho-emotional and cognitive domains represent the outermost dolls that are vulnerable to external damage such as that caused by stroke.

for 4 years in perfect health instead. Another way to interpret this is that the individual would have gained 6 quality adjusted life years $(10-4=6)$ if that stroke was prevented $[4,7]$.

In Jamaica, there has been no study examining how stroke has affected the QOL of its survivors. Studies looking at disease-related QOL have been done in other conditions such as chronic kidney disease and epilepsy. For example, Gayle et al in 2009 looked at the QOL in end-stage renal disease (ESRD) patients using the disease-specific kidney disease quality of life (KDQOL) questionnaire. It showed that Jamaicans had good overall QOL when compared with the KDQOL reference populations [8]. Similarly, Gordon-Perue et al investigated the QOL in Jamaicans with epilepsy, and the results showed that QOL scores were significantly higher than those of other epilepsy populations around the world [9]. It was postulated that the better QOL Jamaicans were experiencing may have been due to the strong religious culture deeply rooted in the Jamaican population with Jamaica allegedly holding the world record for having the most churches per square mile [8]. Similar findings were therefore expected for Jamaican stroke survivors.

Spirituality as an entity has been associated with better QOL outcomes in terminal illnesses such as cancer but few studies have actually looked at this specific entity impacting HRQOL in stroke survivors [10, 11]. In 2004, Giaquinto et al showed that stroke survivors who had higher Royal Free Interview scores (a measure of religiosity) had lower levels of emotional distress. Spirituality has also been affiliated with better physical and mental outcomes in persons living with disabilities [10-13].

In Ibadan Nigeria, a population whose characteristics could be considered to be similar to our own, Dr. Owolabi documents that across culturally distinct stroke cohorts, the physical aspects of QOL decline, while the spiritual aspect is unaffected. He attributed this to the seed of life mode (SOLM) where it is thought that the spiritual components of a man exist at the core of his being, whereas the physical facet is quite superficial leaving it relatively vulnerable to external influences such as physical diseases including stroke; however, the spiritual aspect is so deep that it remains relatively preserved [12] (Fig. 1). It was then theorized that this realm of spirituality could be explored and utilized to improve HRQOL in stroke survivors much like what is done for cancer patients $[11,14$, $15]$.

Landmark studies assessing QOL in stroke such as the North East Melbourne Stroke Incidence Study (NEMESIS) done in Australia, the Auckland Stroke Study done in New Zealand and most recently the China Quality Evaluation of Stroke Care and Treatment (QUEST) have had varying results. Some have observed no effect of stroke on QOL, while others found QOL to be greatly affected by variables such as the degree of physical disability, age, presence of depression and participation in rehabilitation [16-20].

Most of the existing literature implicates increased physical disability as the main factor contributing to decreased QOL in stroke survivors even in cases of mild stroke [12, 15-22]. This is quite significant, particularly as it relates to ischemic stroke where it has been proven that patients who received intravenous recombinant tissue plasminogen activator (rtPA) within the first $3 \mathrm{~h}$ of stroke onset were $30 \%$ more likely to have either negligible physical disability or no disability at all [23]. It can therefore be assumed that these patients will have a better QOL. Currently thrombolytic therapy although accessible remains underutilized in the setting of acute ischemic stroke in Jamaica.

Another key area that calls for greater attention is adherence to treatment regimens for secondary prevention of stroke. Hohmann et al sought to elucidate the impact of pharmaceutical care, a concept that entails a pharmacist working very closely with the patient and the patients' health care provider to refine drug therapy, diminish drug side effects and increase drug adherence, on HRQOL. Overall, it was shown that in- 
tensive pharmaceutical care was able to prevent the decline of most components of HRQOL over the period of a year [24-26]. To date, there has only been one study looking at the QOL in stroke survivors in Jamaica with a specific focus on the effect of aerobic exercise on patients with mild disability [27].They found that aerobic exercise had a favorable effect on QOL in that population.

Overall, HRQOL in stroke is an area that lacks data locally and worldwide but has implications for developing strategies for future planning.

The aims of our study were 1) to compare the HRQOL in a cohort of stroke survivors to that of an AHA population, 2) to determine the association between specific variables and QOL including age, sex, severity of the stroke using the $\mathrm{Na}$ tional Institutes of Health stroke scale (NIHSS), adherence to secondary preventative medication, presence of depression and physical rehabilitation, and finally 3 ) to establish if there was relative preservation of the spiritual aspect of HRQOL in stroke patients.

\section{Methods}

\section{Study population and design}

This study design was a hospital-based case-control study. Data was ascertained from 50 patients with ischemic stroke who were consecutively admitted to the medical floor at the University Hospital of the West Indies (UHWI) in Jamaica between January 2012 and December 2012. Only adults over the age of 16 were allowed to participate. Patients were contacted for recruitment either via telephone or in person at their clinic visit. Interviews took place between April and October 2014 so that patients were between 16 and 28 months post acute stroke. This cohort was compared to an apparently healthy population of 50 adults taken from the hypertension clinic at the UHWI. Similar recruitment measures were used as for the stroke patients. Healthy adults had comparable age and comorbidities to their stroke counterparts. Patients with intraparenchymal hemorrhage, subarachnoid hemorrhage, traumatic hemorrhage, transient ischemic attack (TIA) and those who died during the acute stage of stroke were excluded from the study. The rationale for excluding intra-parenchymal or subarachnoid hemorrhagic stroke includes the fact that they make up the minority of stroke type in Jamaica with previous studies showing a prevalence of $11.1 \%$ and $5.3 \%$, respectively, while ischemic stroke accounted for majority of stroke type with a prevalence of $83.6 \%$ [2]. Also hemorrhagic strokes are associated with higher mortality rates at initial presentation and previous studies have shown no difference in QOL outcomes when comparing these individuals to those with ischemic stroke [28-30]. Therefore, the information gained from including this stroke type would be minimal. Diagnosis of stroke was established by clinical or CT findings by a consultant neurologist. The Trial of Org 10172 in Acute Stroke Treatment (TOAST) classification was utilized to differentiate stroke into different subtypes [31]. Stroke severity was calculated using the NIHSS [23]. Patients' medical records were reviewed for retrospective information about stroke such as NIHSS on admission and CT findings. Data on age, sex, race, marital status, occupation, educational level, co-morbidities, vascular risk factors, specifically hypertension, dyslipidemia, diabetes, smoking, atrial fibrillation and previous stroke were ascertained from medical records and self-reports. The patient health questionnaire 9 (PHQ-9) was used to screen for the presence of depression. It is a well-known screening tool that displays excellent validity, reliability, sensitivity and specificity for the diagnosis of depression $[32,33]$. Persons found to have results suggestive of major depression were referred to the Psychiatry Department at UHWI for further evaluation and management.

The 102-item health-related quality of life in stroke patients (HRQOLISP) questionnaire was used. This questionnaire has been shown to be a culturally diverse instrument with excellent construct validity and reliability in stroke patients $[12,34,35]$. The creator of this questionnaire, Dr. M. Owolabi granted permission for its use in this study via email correspondence. The questionnaire itself is divided into seven domains: physical, psychoemotional, cognitive, ecosocial interaction, soul, spirit and spiritual interaction domains. These seven domains are then separated into two main spheres. The physical sphere consists of the physical, psychoemotional, cognitive and ecosocial interaction. The spiritual sphere encompasses the soul, spirit and spiritual interaction domains. Scores for each domain are then summed together with a maximum score of 100 for each. The questionnaire was administered either as a face to face interview or via telephone. The average time for completion in previous studies was $27 \mathrm{~min}$; however, in this study, average completion time was $45 \mathrm{~min}$. Patients were allowed to complete the questionnaire in multiple sittings. The HRQOLISP measure has already been shown to be applicable in settings where the stroke victims may not be able to respond because of severe disability such as aphasia or concentration; in such cases, a proxy (usually a family member or care taker) could act as a respondent. Proxies were accepted as reliable if they were either living with the patient or were closely related to the patient. In general, most tools however do show that proxy respondents tend to rate the QOL as poorer than the patient would rate themselves $[16,18,25,26,36]$. In order to prevent selection bias that may arise from excluding the particular population of stroke patients that have difficulty communicating with the interviewer, proxies were used. Written consent was ascertained from all participants. This study was approved by the University of the West Indies (UWI) ethics committee.

\section{Statistical analysis}

The sample size was calculated as follows; in 2011 the medical records department at the UHWI coded 87 patients as being admitted for ischemic stroke. In 2012, 108 patients were coded as being admitted for ischemic stroke. Taking the average of these two numbers, a target population size was 97.5. Using the Raosoft sample size calculator accepting a margin of error of $5 \%$, a confidence interval of $95 \%$ and a response distribution of $50 \%$, then the recommended sample size is 78 . The 
Table 1. Baseline Demographics and Clinical Information on Stroke Survivors and Apparently Healthy Adults (AHAs)

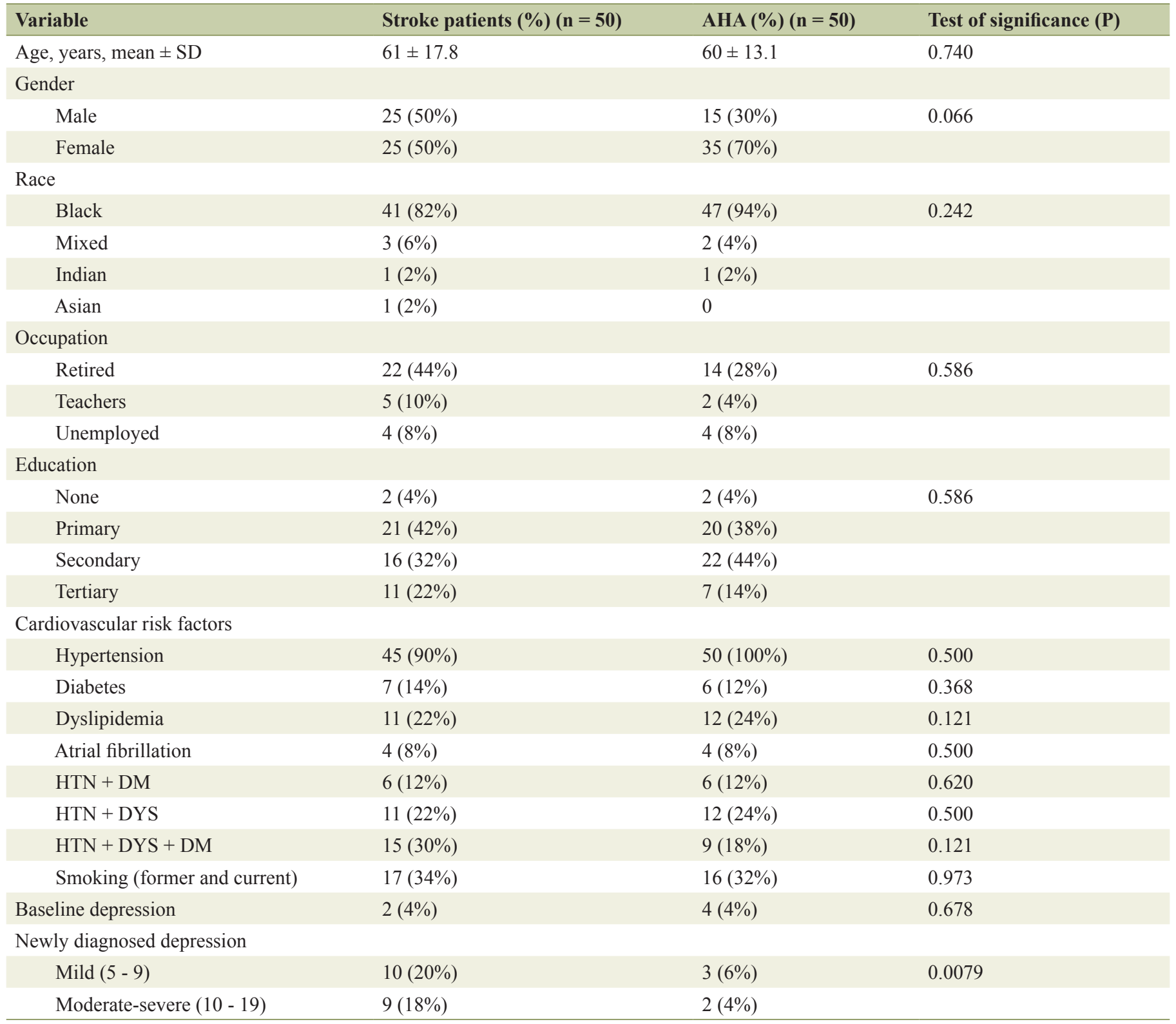

minimum sample size required for each group to detect a difference in QOL scores at $80 \%$ power was 20 .

Demographic information for the two groups were analyzed using both SPSS and Stata. Non-categorical variables were expressed as means with standard deviations. Differences between means of the stroke patients versus the healthy adults were compared using the Student's $t$-test and two-way ANOVA.

The Pearson test was used to correlate continuous variables with other continuous variables and the Spearman test was used to compare categorical with continuous variables. A value of $\mathrm{P}<0.05$ was deemed statistically significant. HRQOLISP scores were generated by Likert's method where each successive option was treated as moving up or doing better in the scale. This was done after recalibrating the items such that a higher score was always indicative of a better QOL. Each domain had a maximum score of 100 . Each sphere was calculated by averaging the scores in the respective domains. The total HRQOLISP score was attained by finding the mean of the all seven domains for each participant.

\section{Results}

Of the 108 patients that were coded as being admitted for acute ischemic stroke during the year 2012, 38 (35\%) died, $17(15.7 \%)$ could not be located for follow-up, two $(1.8 \%)$ migrated and one $(0.9 \%)$ had refused to participate. This left 


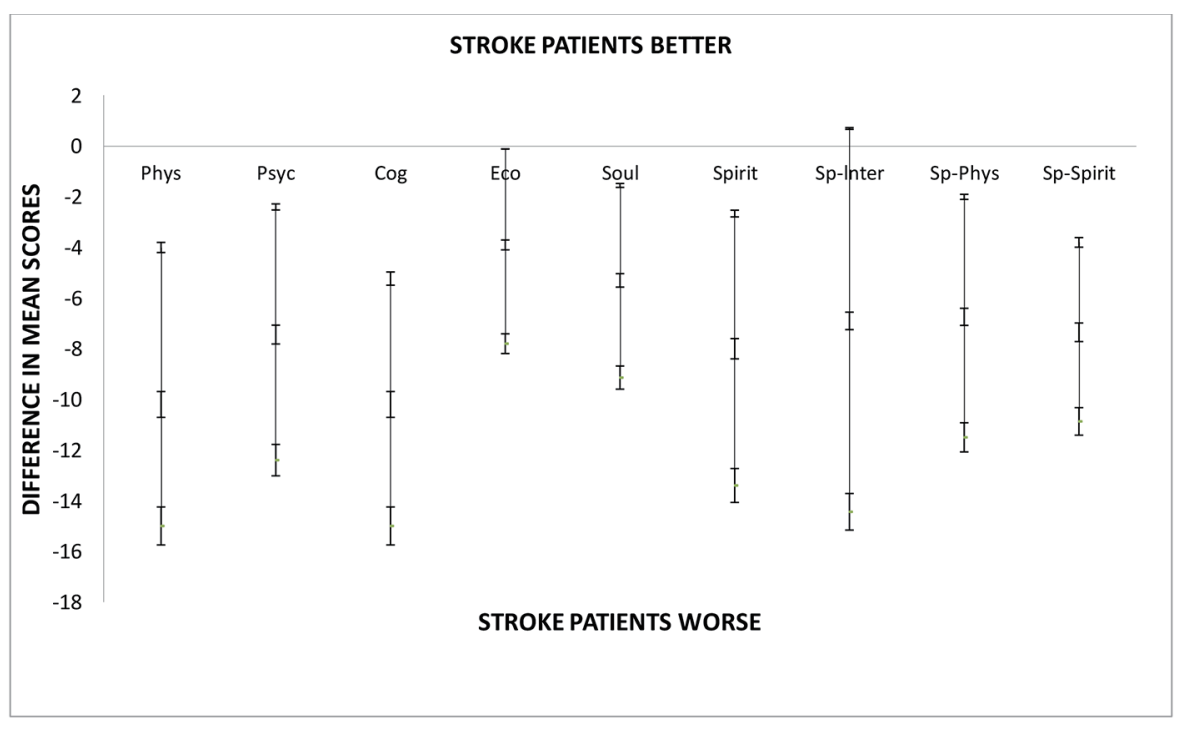

Figure 2. Difference between mean HRQOLISP scores in each domain of QOL for stroke patients and apparently healthy adults at the UHWI. As shown stroke patients were doing significantly worse in all domains except the spiritual interaction domain. SpSpirit: spiritual sphere; Sp-Phys: physical sphere; Splnt: spiritual interaction domain; Spirit: spirit domain; Phys: physical domain; Psyc: psycho-emotional domain; Cog: cognitive domain; Psyc: psycho-emotional domain; Phys: physical domain.

$50(46 \%)$ patients available for interview. Patients' baseline demographic information and co-morbidities are summarized in Table 1. There was no statistical difference between the stroke group and the AHAs with regard to age, race, educational status and traditional risk factors for stroke. Within the stroke population, the mean age was $61 \pm 17.8$ years .Of the 45 patients with stroke that had hypertension, $44(97 \%)$ reported that they had been prescribed antihypertensive medication; however, only 32 (73\%) reported adherence with same. More devastatingly, only $26(62 \%)$ reported compliance with anti-platelet medication. Fifteen (30\%) stroke patients also reported they had suffered a previous stroke the timeline of which was not ascertained. The most common cardiovascular risk factor for both groups was hypertension. This was trailed by former or current cigarette smoking in both groups (Table 1).

\section{HRQOL scores}

HRQOLISP scores for the stroke survivors are shown in Figure 2. Between 16 and 28 months post stroke, the HRQOL in stroke survivors was markedly worse than that of the apparently healthy group in all domains except the spiritual interaction domain (Table 2). This difference was most noticeable in the physical and cognitive domains $(\mathrm{P}=0.0003$ and $\mathrm{P}=0.0001$, respectively). When comparing the spiritual sphere scores between the two groups, the stroke patients scored significantly lower than the AHAs $(\mathrm{P}=0.005)$ (Fig. 2). Older age, female sex, large vessel, stroke subtype (Fig. 3), physical rehabilita-

Table 2. Difference Between Mean HRQOL in Stroke Survivors Versus Apparently Healthy Adults (AHAs)

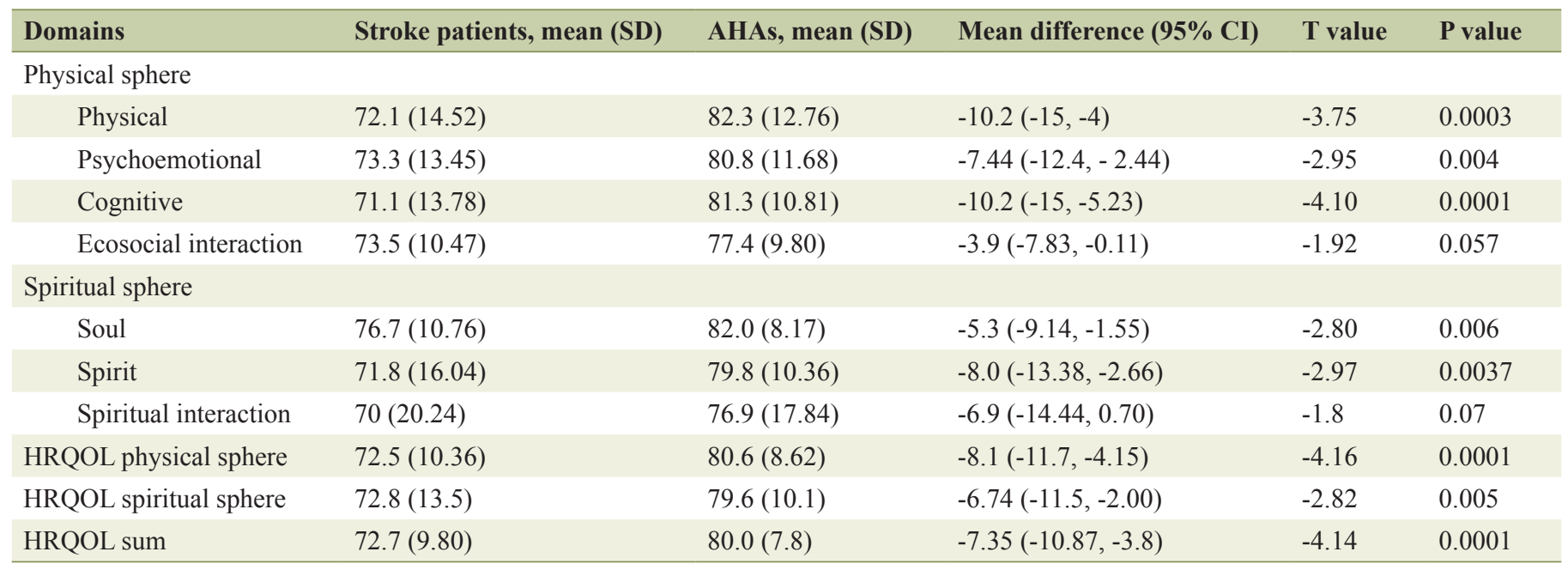




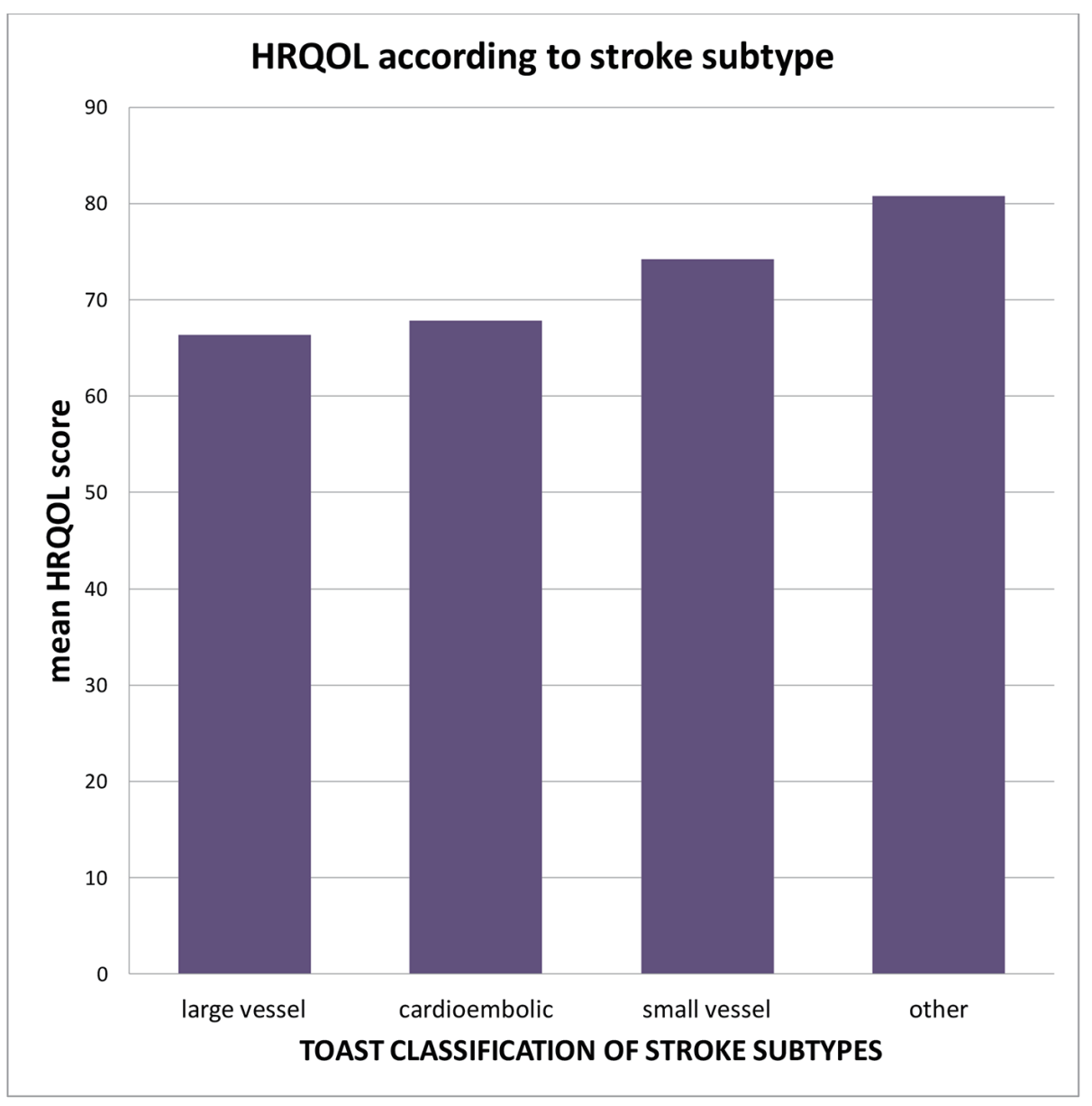

Figure 3. Mean HRQOL scores within each subtype of ischemic stroke. Large vessel and cardioembolic strokes were associated with lower HRQOL mean score when compared to small vessel arteriosclerotic subtype.

tion for less than 4 weeks and non-compliance with secondary prevention medication were associated with worse HRQOL but these did not meet statistical significance. Variables that were found to significantly worsen HRQOL were 1) greater stroke severity as assessed by NIHSS at admission, 2) higher PHQ-9 scores with presence of depression, 3) previous stroke, and 4) proxy respondents with proxies rating HRQOL for the stroke patients much lower than self-responders (Fig. 4).

\section{NIHSS}

Prior studies have used the NIHSS to divide stroke severity into mild, moderate and severe categories. Scores $<8$ represent mild stroke, 8 - 16 represent moderate stroke and scores of $>17$ are deemed severe stroke $[23,37]$. In our study, the mean NIHSS was $5.2 \pm 4.9$. There were $41(82 \%)$ cases of mild stroke, six (12\%) cases of moderate stroke and three $(6 \%)$ cases of severe stroke. The mean QOL declined drastically with each increase in the stroke severity category with persons having a mild stroke having an average QOL score of 74 while persons with severe stroke had a mean QOL score of $65.2(\mathrm{P}$ $=0.03$ ) (Table 3 and Fig. 4). Using NIHSS as a continuous variable, there was a linear decline in mean HRQOL as NIHSS increased (Fig. 5). Large vessel stroke subtype was also significantly associated with higher NIHSS $(\mathrm{P}=0.004)$ (Fig. 6). Female sex correlated with higher NIHSS scores but this was not found to be statistically significant.

\section{PHQ-9 scores}

The PHQ-9, as mentioned previously, exhibits excellent validity and reliability $[32,33]$. It is very easy to administer and interpret. A PHQ-9 score $\geq 10$ has been shown to have a specificity of $88 \%$ and a sensitivity of $88 \%$ for major depression. In our study, nine (18\%) of patients with stroke had a PHQ-9 score $\geq 10$, suggestive of moderate to severe depression compared to just two (4\%) in the apparently healthy group. This difference was thought to be statistically significant $(\mathrm{P}<0.01)$ (Fig. 7). Overall $19(38 \%)$ of the stroke patients had at least mild depression compared to five $(10 \%)$ in the apparently healthy population (Table 1). Cognitive impairment, as assessed by the cognitive domain of the HRQOLISP, was significantly correlated with depression $(\mathrm{P}<0.01)$. Greater stroke severity and female sex were among several other variables found to be 


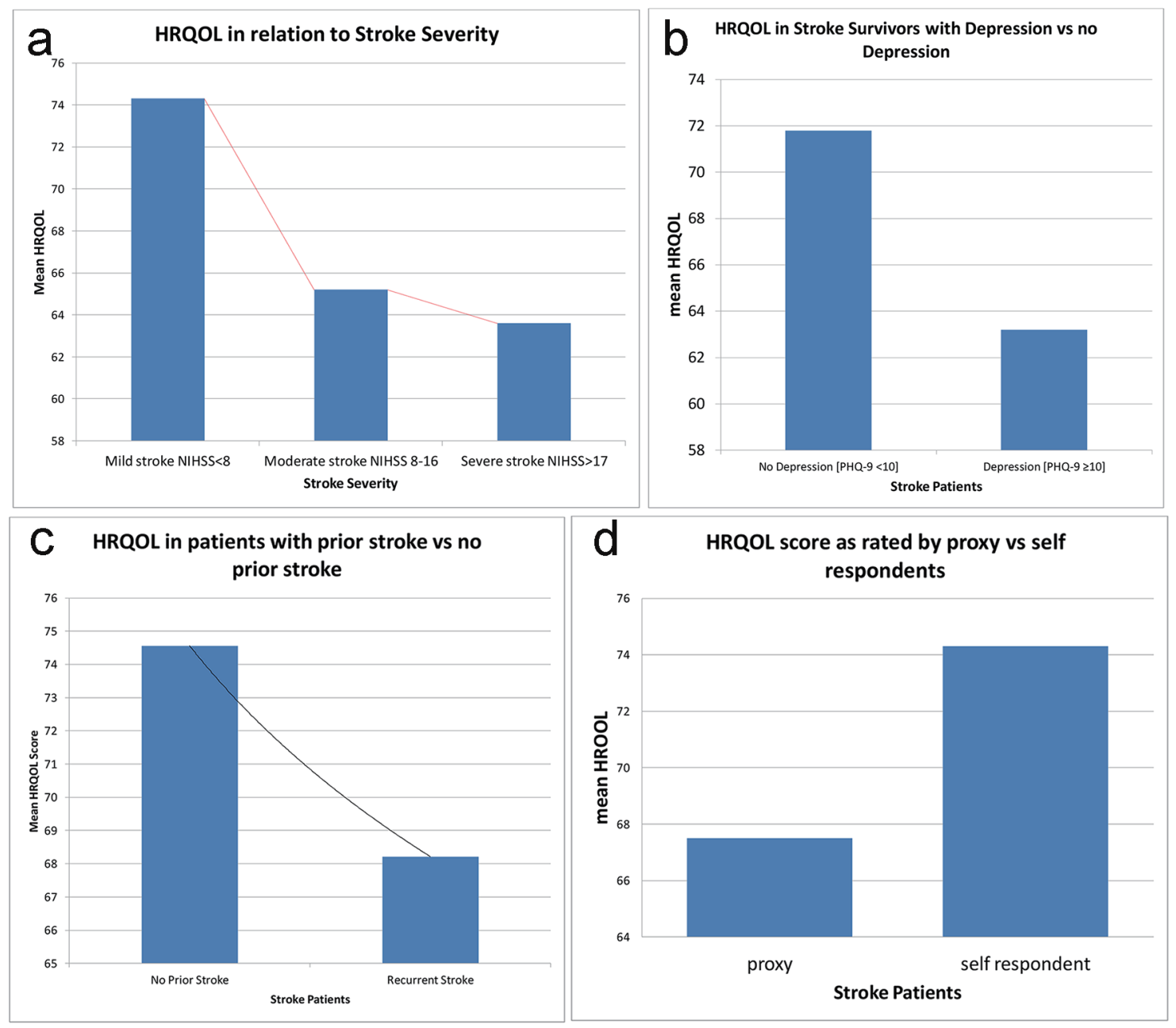

Figure 4. Variables that were significantly associated with worse HRQOL in stroke patients at UHWI. (a) Decreasing mean HRQOL with increasing stroke severity as measured by NIHSS $(P=0.0046)$. (b) Decrease in mean QOL if depression was present $(P=0.0040)$. (c) Decrease in mean $Q O L$ if the patient had a prior stroke $(P<0.05)$. (d) Decrease in mean $Q O L$ if the HRQOLISP was being completed by a proxy $(P<0.05)$.

associated with depression but these were not shown to be statistically significant. Presence of depression greatly decreased HRQOL in our stroke cohort of patients.

\section{Discussion}

Despite the relatively high prevalence of stroke in Jamaica, limited data exist on HRQOL outcomes in our stroke survivors $[1,27]$. The results of this study have partially addressed this void in the literature. It has shown that stroke survivors, at least 16 months post stroke, have significantly reduced HRQOL scores in all domains except the spiritual interaction domain. This decline in HRQOL is only expected to worsen as time progresses. The Northern Manhattan study highlights the linear relationship between the two, showing that disability increases with time even when there is no evidence of stroke recurrence. The follow-up for the NEMISIS study went on to show that even 7 years post stroke, survivors showed persis- tently low HRQOL $[21,38]$.

It is surprising that we observed no preservation of the spiritual sphere of HRQOL in our stroke cohort as was observed in Ibadan, Nigeria, given that previous QOL studies done in Jamaica for other disease states used the spirituality of Jamaicans to account for their relatively preserved QOL. When comparing mean HRQOL scores between Ibadan and Jamaica, Jamaicans were experiencing significantly lower means in the spirit domain (difference of -7.1) and the spiritual interaction domain (difference of -6.8). The reason for this decline specifically in stroke as opposed to other diseases in Jamaica could be looked at in future studies.

Our study has reiterated the fact that even in cases of mild stroke with minimal disability, stroke survivors were still experiencing poor HRQOL when compared to their healthy counterparts. This difference was not explained by co-morbidities as these were not different between the two groups. It is a phenomenon that has been repeatedly observed in the literature with studies showing that even in TIAs, where physi- 
Table 3. Correlation of Stroke-Specific Variables With Mean HRQOL

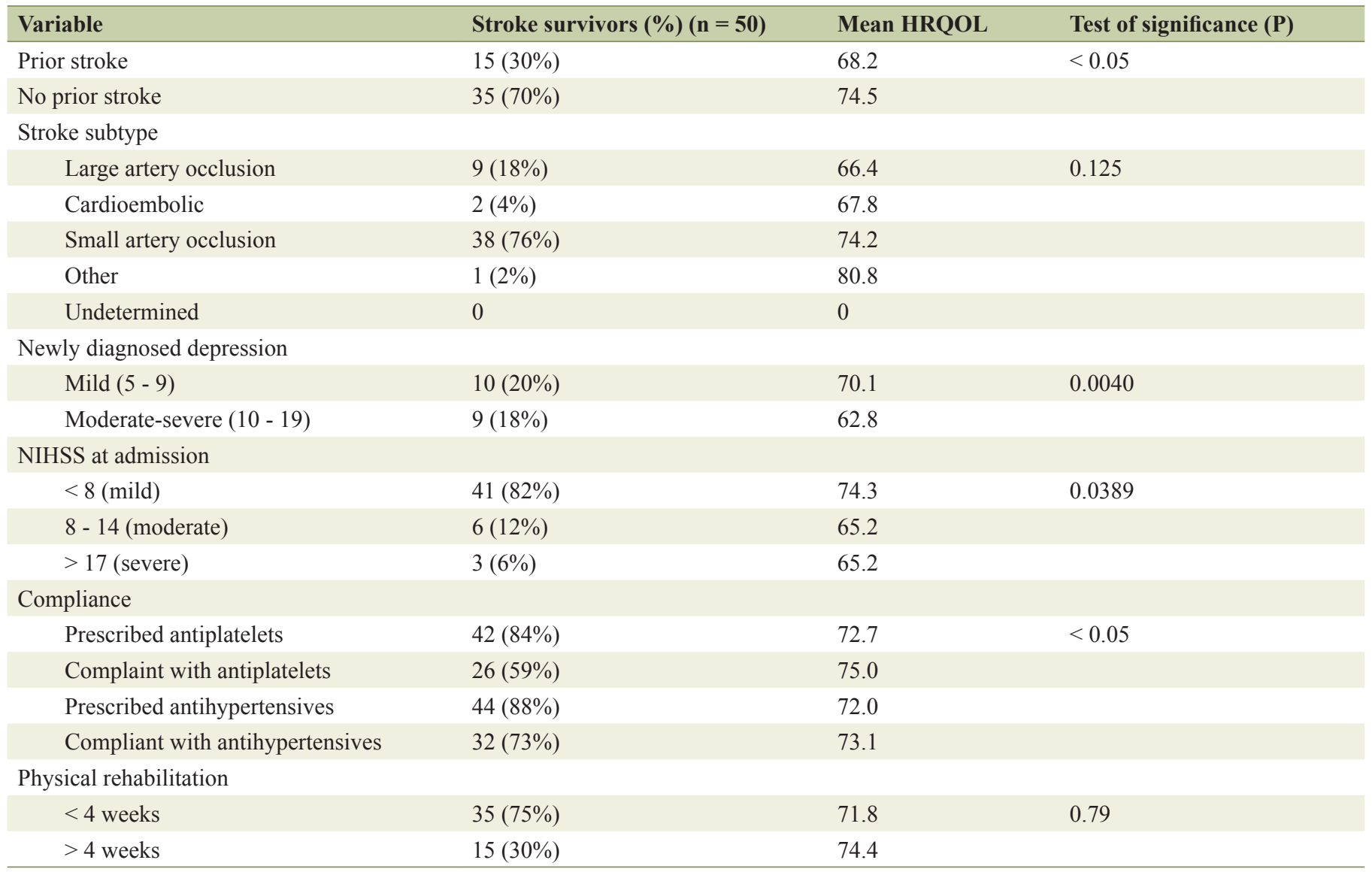

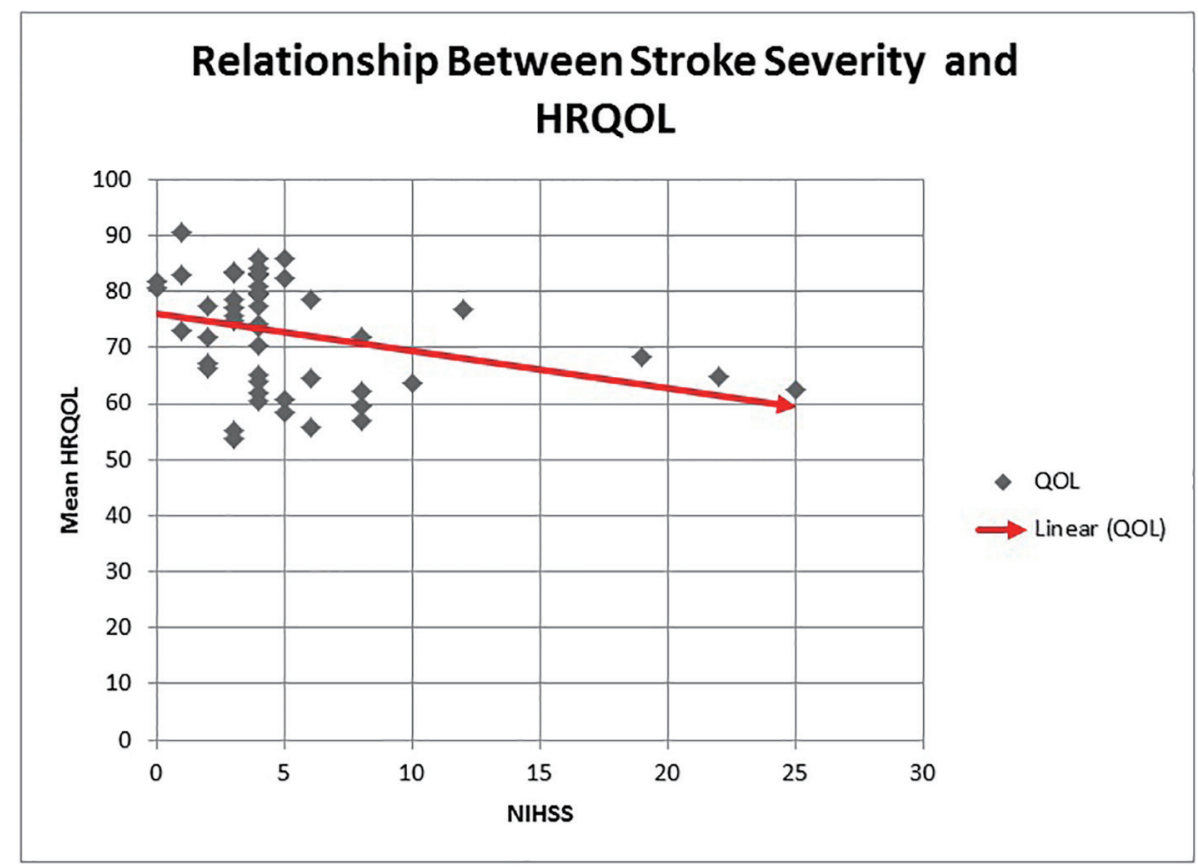

Figure 5. The relationship between stroke severity and HRQOL. An increase in stroke severity as measured by the admission NIHSS is associated with a linear decline in mean HRQOL. 


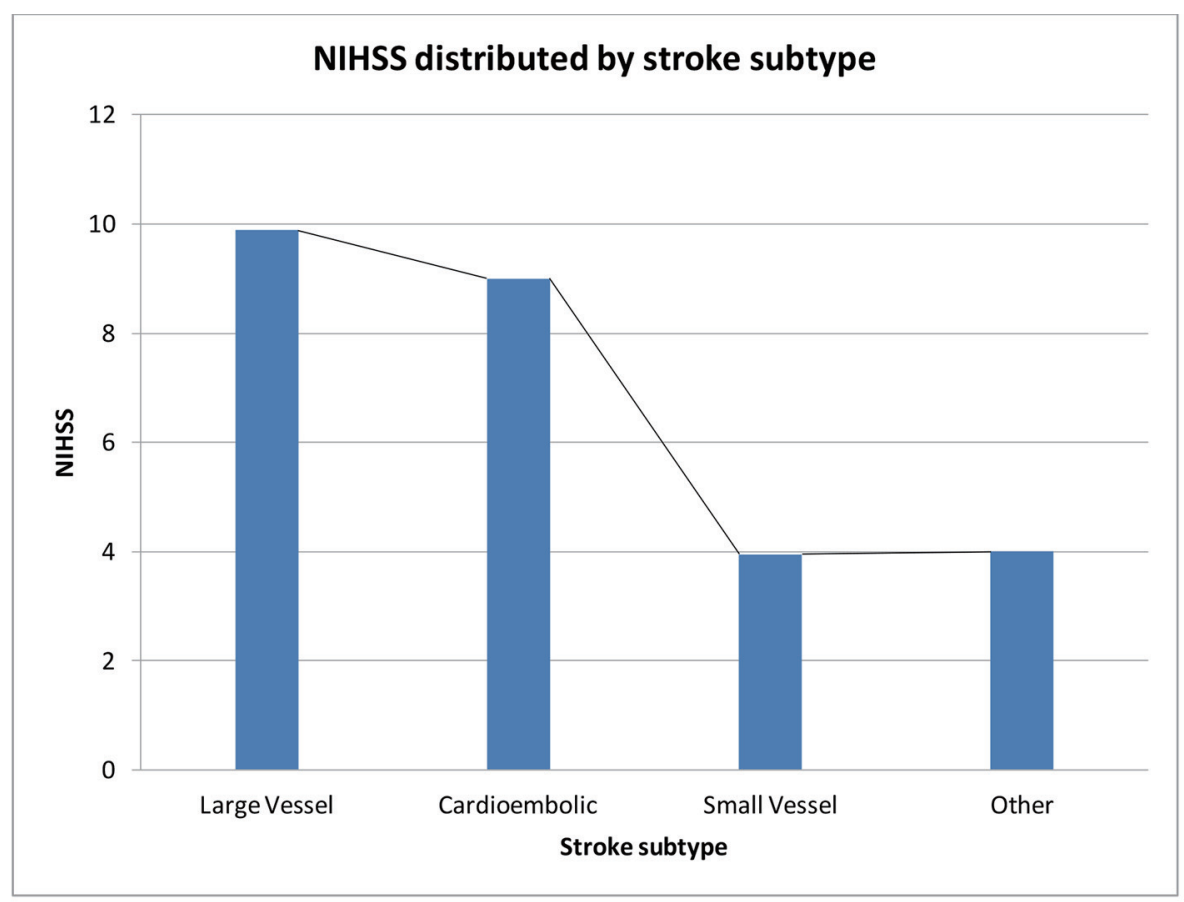

Figure 6. Mean NIHSS within each stroke subtype using the TOAST classification for ischemic stroke. Patients with large artery occlusion and cardioembolic subtypes had significantly higher NIHSS at admission than patients with small artery occlusion subtype of stroke.

cal disability has completely resolved, HRQOL continues to be less than population norms $[19,38]$. Some have attributed this to the fact that approximately $30 \%$ of patients with TIA and mild stroke have CT findings showing atherosclerotic changes in small vessels or white matter disease. These white matter changes although often asymptomatic, are implicated in cognitive decline, development of mood disorders and poor HRQOL primarily due to a decline in mental functioning [3941]. This would be in keeping with our study where the cognitive domain was one of the two domains most significantly

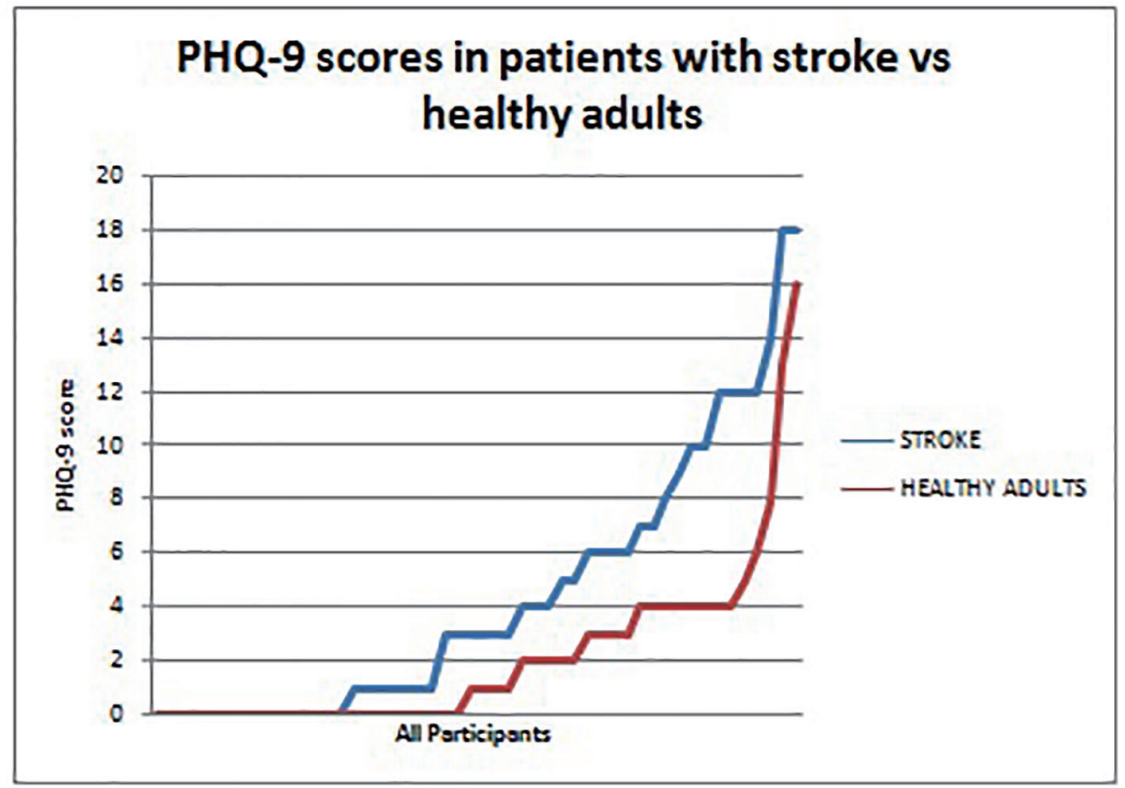

Figure 7. PHQ-9 scores in patients with stroke versus healthy adults. Patients with stroke (blue) consistently had higher PHQ-9 scores than the healthy adults (red). This represents the greater prevalence of depression among stroke survivors when compared to a reference healthy population. 
affected by stroke $(P=0.0001)$. White matter changes could also account for the high prevalence of depression as it has been proposed that these changes interrupt the cortical- subcortical connections regulating mood as seen on diffusion tensor imaging [41]. It would be interesting to revisit CT or MRI in our stroke participants to document the presence or absence of white matter changes in these individuals to explain poor HRQOL despite minimal disability and is perhaps a task that can be tackled in the future.

White matter changes could also account for the lower QOL scores in our healthy control group drafted from the hypertension clinic versus healthy controls used in other countries. It has been shown that elderly patients with hypertension have 10 times the risk of developing white matter changes as compared to controls of similar age [40].

Studies have also shown that the risk of developing depression after mild stroke or TIA greatly exceeds that of the general population. This depression can in turn contribute to poor HRQOL [42]. Hommel et al showed that even in mild stroke, $68 \%$ of patients complained of significant disruption in social functioning such as returning to work and maintaining close relationships. Loss of concentration, fatigue, irritability and perceived disability were some of the factors used to explain this disruption. Cognitive training was suggested as an intervention that could be useful in this scenario and may warrant prospective studies here in our stroke survivors [43, 44]. The aforementioned becomes exceedingly important as $82 \%$ of the stroke population in our study had what would be considered mild stroke using the admitting NIHSS.

In our study, the variables that significantly correlated with lower HRQOL scores were greater stroke severity, presence of depression, recurrent stroke and proxy respondents. These variables are consistently associated with worse QOL outcomes in stroke cohorts all over the world [15-21]. Women, age greater than 75 , large vessel or anterior circulation stroke type, those requiring assistance with activities of daily living and those residing in institutions are other common determinants of poor HRQOL outcomes; however, these were not found to be statistically significant in our study, perhaps due to the relatively small sample size.

Proxy responders tended to rate stroke survivors with worse QOL scores than if the stroke patients were to respond themselves. This effect has been reproduced and observed in multiple stroke-related QOL studies. This may be due to the fact that only the most disabled patients would be unable to complete the questionnaire by themselves and so naturally would have worse scores [12, 17, 36].

In terms of stroke severity, our study showed a linear relationship between NIHSS and decline in mean HRQOL (Fig. 5). Adams et al found that for every additional point increase in the NIHSS, the chance of a better outcome post stroke decreased by an impressive $24 \%$ at 7 days and $17 \%$ at 3 months [31]. This should hopefully spark efforts to reduce patients initial NIHSS in the acute setting of ischemic stroke with greater use of intravenous rtPA. Unfortunately, high costs of rtPA and lack of public awareness about acute stroke management promote the underutilization of this intervention. Many Jamaicans present 3 - 4 days after stroke onset greatly restricting the use of this invaluable tool. National policies involving public edu- cation would have to be developed to tackle this particular issue.

Depression is the most common psychiatric condition occurring post stroke, with the prevalence of major depression being quoted at $15-20 \%$ in the acute setting. Some studies found rates as low as $9 \%$ while others quoted rates as high as $50 \%[13,19,20,45]$. Although the prevalence of major depression tends to decrease as time progresses, minor depression often persists [45]. Despite its high prevalence, post stroke depression (PSD) remains vastly underdiagnosed. Husseini et al demonstrated that among patients with persistent PSD after 1 year, $67 \%$ were not placed on antidepressants despite this condition being responsive to same [42]. Factors associated with PSD include greater stroke disability, dependence in activities of daily living, inability to work, greater cognitive decline, dysphasia, left anterior brain lesion and living alone [42, 45].

In our stroke survivors, $19(38 \%)$ were found to have symptoms of depression, be it mild, moderate or severe, and nine (18\%) had PHQ-9 scores placing them in the severe depression category. None of these patients had been previously evaluated or diagnosed with depression. Conversely, just five $(10 \%)$ of the AHAs were found to have symptoms of depression and two (4\%) were found to have PHQ-9 scores placing them in the severe depression category. It follows therefore that stroke survivors should be screened regularly for symptoms of depression at their follow-up appointments. Many persons with mild stroke however are discharged from the hospital setting to the care of a general practitioner. Efforts should be made to educate this subset of doctors on the high prevalence and poor outcomes associated with PSD so that appropriate screening, referrals and interventions can occur at follow-up visits.

When looking at secondary stroke prevention, $73 \%$ of stroke survivors were adherent to their antihypertensive, while just $62 \%$ were adherent to their anti-platelet therapy. Fifteen $(30 \%)$ of the strokes were recurrent stroke. This figure is similar to that of $27 \%$ found in Trinidad, but higher than the $18 \%$ reported in the US $[5,46]$. Recurrent stroke was significantly associated with lower HRQOL scores in our study. The benefit of anti-platelet and antihypertensive therapy in preventing recurrent stroke has been well documented [47, 48]. Antihypertensive drugs have also been shown to halt or delay the progression of white matter changes which we suspect many of our participants had [49]. Therefore, follow-up studies looking at reasons for non-adherence in this vulnerable population, be it side effects or cost, become critical. The concept of intensive pharmaceutical care is something that could possibly be adopted by the pharmacist at UHWI in order to optimize this very important aspect of care. As mentioned earlier, intensive pharmaceutical care has been shown to prevent deterioration of most constituents of HRQOL 1 year post stroke [24]. Although our findings found a correlation between better HRQOL and adherence with medication, this was not found to be statistically significant. This may be due to the small sample size or it may suggest that there is another variable unaccounted for. The AVAIL study also found no correlation between persistence with secondary preventative medication and better QOL outcomes. The lack of correlation observed may have been due to the study's use of a generic QOL tool. These conflicting results 
leave unanswered questions that encourage further studies to be done locally in this area $[25,26]$.

Lastly, although this was not an objective of the study, mortality rates were disturbingly high enough to warrant discussion. We found that $35 \%$ of patients with stroke had died by the time of follow-up 1 - 2 years later. Information was not ascertained as to their cause of death to determine if death was directly related to their stroke. If it were, it would indicate that our stroke population has a particularly high mortality rate when compared to both our neighbours in the Caribbean and the global village with Guadeloupe having a mortality of $27.8 \%$ one year post stroke, the US having a rate of $9-23 \%$ depending on age and the Netherlands having rates as low as $1.2-2.9 \%$ one year post stroke $[5,50]$.

\section{Conclusions}

This study provides us with much needed insight into the factors affecting HRQOL outcomes in Jamaican stroke survivors. It is hoped that the information gained will ignite institutional and national policies targeting physical disability, depression and stroke recurrence in this very vulnerable population.

\section{Study limitations}

QOL was assessed using a cross-sectional method meaning that patients were being assessed at different times during their stroke recovery period. QOL tends to change after years or even months of stroke. It would have been ideal to interview everyone at a specific interval post stroke, for instance 1 year, as was done in previous studies; however, due to limited time for data collection, this was not possible.

The HRQOLISP though a holistic and thorough tool remains very time consuming with an average completion time of $45 \mathrm{~min}$. This limits its use for other studies. The lack of standardization of stroke-related QOL instruments globally makes it difficult to compare. However with the use of this tool we were at least able to directly compare our cohort to those in Nigeria, and Germany.

\section{Acknowledgments}

Medical students: Ronique Gordon, Kimberly Deenah and Nurses: Heather Phillips ,Priscilla Jamieson.

\section{Competing Interests}

There are no competing interests.

\section{Author Contributions}

Kerice Pinkney, Sandra Pinkney, Maudel Charlton, and Priscilla Jamieson: data collection. Nollis Williams, Kimberly
Deenah, and Ronique Gordon: data calculation. Heather Phillips: patient recruitment.

\section{References}

1. Ferguson TS, Younger N, Morgan ND. Self-reported prevalence of heart attacks and strokes in Jamaica: a cross-sectional study. The Jamaica Health and Lifestyle Survey 2007-2008. Research Reports in Clinical Cardiology. 2010;1:23-31.

2. West WM, Younger N, Brady-West D, Gilbert DT, Char $\mathrm{G}$, Barton EN. Stroke subtypes on imaging in a Jamaican population: a hospital-based study. West Indian Med J. 2009;58(3):261-264.

3. Lozano R, Naghavi M, Foreman K, Lim S, Shibuya K, Aboyans V, Abraham J, et al. Global and regional mortality from 235 causes of death for 20 age groups in 1990 and 2010: a systematic analysis for the Global Burden of Disease Study 2010. Lancet. 2012;380(9859):2095-2128.

4. Tengs TO, Yu M, Luistro E. Health-related quality of life after stroke a comprehensive review. Stroke. 2001;32(4):964-972.

5. Go AS, Mozaffarian D, Roger VL, Benjamin EJ, Berry JD, Blaha MJ, Dai S, et al. Heart disease and stroke statistics--2014 update: a report from the American Heart Association. Circulation. 2014;129(3):e28-e292.

6. Development of the World Health Organization WHOQOL-BREF quality of life assessment. The WHOQOL Group. Psychol Med. 1998;28(3):551-558.

7. Tseng MC, Lin HJ. Health-related quality of life after stroke: review of the literature and implications for future research. Acta Neurol Taiwan. 2007;16(1):7-12.

8. Gayle F, Soyibo AK, Gilbert DT, Manzanares J, Barton EN. Quality of life in end stage renal disease: a multicentre comparative study. West Indian Med J. 2009;58(3):235242.

9. Gordon-Perue G, Gayle F, Fraser R, Ali A. Quality of life of patients with epilepsy living in Kingston, Jamaica. Epilepsy Behav. 2011;21(1):23-26.

10. Balboni TA, Paulk ME, Balboni MJ, Phelps AC, Loggers ET, Wright AA, Block SD, et al. Provision of spiritual care to patients with advanced cancer: associations with medical care and quality of life near death. J Clin Oncol. 2010;28(3):445-452.

11. Phelps AC, Lauderdale KE, Alcorn S, Dillinger J, Balboni MT, Van Wert M, Vanderweele TJ, et al. Addressing spirituality within the care of patients at the end of life: perspectives of patients with advanced cancer, oncologists, and oncology nurses. J Clin Oncol. 2012;30(20):25382544.

12. Owolabi MO. Impact of stroke on health-related quality of life in diverse cultures: the Berlin-Ibadan multicenter international study. Health Qual Life Outcomes. 2011;9:81.

13. Giaquinto S, Spiridigliozzi C, Caracciolo B. Can faith protect from emotional distress after stroke? Stroke. 2007;38(3):993-997.

14. Schreiber JA, Brockopp DY. Twenty-five years later- 
-what do we know about religion/spirituality and psychological well-being among breast cancer survivors? A systematic review. J Cancer Surviv. 2012;6(1):82-94.

15. Ellis C, Grubaugh AL, Egede LE. Factors associated with SF-12 physical and mental health quality of life scores in adults with stroke. J Stroke Cerebrovasc Dis. 2013;22(4):309-317.

16. Sturm JW, Donnan GA, Dewey HM, Macdonell RA, Gilligan AK, Srikanth V, Thrift AG. Quality of life after stroke: the North East Melbourne Stroke Incidence Study (NEMESIS). Stroke. 2004;35(10):2340-2345.

17. Hackett ML, Duncan JR, Anderson CS, Broad JB, Bonita R. Health-related quality of life among long-term survivors of stroke : results from the Auckland Stroke Study, 1991-1992. Stroke. 2000;31(2):440-447.

18. Delcourt C, Hackett M, Wu Y, Huang Y, Wang J, Heeley E, Wong L, et al. Determinants of quality of life after stroke in China: the ChinaQUEST (QUality Evaluation of Stroke care and Treatment) study. Stroke. 2011;42(2):433438.

19. Klocek M. Quality of Life After Stroke. In Health-Related Quality of Life in Cardiovascular Patients. Springer. 2013:103-117.

20. Carod-Artal J, Egido JA, Gonzalez JL, Varela de Seijas E. Quality of life among stroke survivors evaluated 1 year after stroke: experience of a stroke unit. Stroke. 2000;31(12):2995-3000.

21. Leach MJ, Gall SL, Dewey HM, Macdonell RA, Thrift AG. Factors associated with quality of life in 7-year survivors of stroke. J Neurol Neurosurg Psychiatry. 2011;82(12):1365-1371.

22. Hommel M, Trabucco-Miguel S, Joray S, Naegele B, Gonnet N, Jaillard A. Social dysfunctioning after mild to moderate first-ever stroke at vocational age. J Neurol Neurosurg Psychiatry. 2009;80(4):371-375.

23. Tissue plasminogen activator for acute ischemic stroke. $\mathrm{N}$ Engl J Med. 1995;333(24):1581-1587.

24. Hohmann C, Radziwill R, Klotz JM, Jacobs AH. Research Health-Related Quality of Life after Ischemic Stroke: The Impact of Pharmaceutical Interventions on Drug Therapy (Pharmaceutical Care Concept). 2010.

25. Bushnell CD, Olson DM, Zhao X, Pan W, Zimmer LO, Goldstein LB, Alberts MJ, et al. Secondary preventive medication persistence and adherence 1 year after stroke. Neurology. 2011;77(12):1182-1190.

26. Bushnell C, Zimmer L, Schwamm L, Goldstein LB, Clapp-Channing N, Harding T, Drew L, et al. The Adherence eValuation After Ischemic Stroke Longitudinal (AVAIL) registry: design, rationale, and baseline patient characteristics. Am Heart J. 2009;157(3):428-435 e422.

27. Gordon CD, Wilks R, McCaw-Binns A. Effect of aerobic exercise (walking) training on functional status and health-related quality of life in chronic stroke survivors: a randomized controlled trial. Stroke. 2013;44(4):11791181 .

28. Christensen MC, Mayer S, Ferran JM. Quality of life after intracerebral hemorrhage: results of the Factor Seven for Acute Hemorrhagic Stroke (FAST) trial. Stroke. 2009;40(5):1677-1682.
29. de Haan RJ, Limburg M, Van der Meulen JH, Jacobs HM, Aaronson NK. Quality of life after stroke. Impact of stroke type and lesion location. Stroke. 1995;26(3):402408.

30. Lee HY, Hwang JS, Jeng JS, Wang JD. Quality-adjusted life expectancy (QALE) and loss of QALE for patients with ischemic stroke and intracerebral hemorrhage: a 13year follow-up. Stroke. 2010;41(4):739-744.

31. Adams HP, Jr., Davis PH, Leira EC, Chang KC, Bendixen BH, Clarke WR, Woolson RF, et al. Baseline NIH Stroke Scale score strongly predicts outcome after stroke: A report of the Trial of Org 10172 in Acute Stroke Treatment (TOAST). Neurology. 1999;53(1):126-131.

32. Williams LS, Brizendine EJ, Plue L, Bakas T, Tu W, Hendrie H, Kroenke K. Performance of the PHQ-9 as a screening tool for depression after stroke. Stroke. 2005;36(3):635-638.

33. Kroenke K, Spitzer RL, Williams JB. The PHQ-9: validity of a brief depression severity measure. J Gen Intern Med. 2001;16(9):606-613.

34. Owolabi MO. Which is more valid for stroke patients: generic or stroke-specific quality of life measures? Neuroepidemiology. 2010;34(1):8-12.

35. Ojo Owolabi M. HRQOLISP-26: A Concise, Multiculturally Valid, Multidimensional, Flexible, and Reliable Stroke-Specific Measure. ISRN Neurol. 2011;2011:295096.

36. Sneeuw KC, Aaronson NK, de Haan RJ, Limburg M. Assessing quality of life after stroke. The value and limitations of proxy ratings. Stroke. 1997;28(8):1541-1549.

37. Briggs DE, Felberg RA, Malkoff MD, Bratina P, Grotta JC. Should mild or moderate stroke patients be admitted to an intensive care unit? Stroke. 2001;32(4):871-876.

38. Dhamoon MS, Moon YP, Paik MC, Boden-Albala B, Rundek T, Sacco RL, Elkind MS. Quality of life declines after first ischemic stroke. The Northern Manhattan Study. Neurology. 2010;75(4):328-334.

39. Dhamoon MS, McClure LA, White CL, Lau H, Benavente O, Elkind MS. Quality of life after lacunar stroke: the Secondary Prevention of Small Subcortical Strokes study. J Stroke Cerebrovasc Dis. 2014;23(5):1131-1137.

40. van Swieten JC, Geyskes GG, Derix MM, Peeck BM, Ramos LM, van Latum JC, van Gijn J. Hypertension in the elderly is associated with white matter lesions and cognitive decline. Ann Neurol. 1991;30(6):825-830.

41. Brookes RL, Herbert V, Lawrence AJ, Morris RG, Markus HS. Depression in small-vessel disease relates to white matter ultrastructural damage, not disability. Neurology. 2014;83(16):1417-1423.

42. El Husseini N, Goldstein LB, Peterson ED, Zhao X, Pan W, Olson DM, Zimmer LO, et al. Depression and antidepressant use after stroke and transient ischemic attack. Stroke. 2012;43(6):1609-1616.

43. Belleville S. Cognitive training for persons with mild cognitive impairment. Int Psychogeriatr. 2008;20(1):5766.

44. Willis SL, Tennstedt SL, Marsiske M, Ball K, Elias J, Koepke KM, Morris JN, et al. Long-term effects of cognitive training on everyday functional outcomes in older 
adults. JAMA. 2006;296(23):2805-2814.

45. Chemerinski E, Levine SR. Neuropsychiatric disorders following vascular brain injury. Mt Sinai J Med. 2006;73(7):1006-1014.

46. Mahabir D, Bickram L, Gulliford MC. Stroke in Trinidad and Tobago: burden of illness and risk factors. Rev Panam Salud Publica. 1998;4(4):233-237.

47. Diener HC, Cunha L, Forbes C, Sivenius J, Smets P, Lowenthal A. European Stroke Prevention Study. 2. Dipyridamole and acetylsalicylic acid in the secondary prevention of stroke. J Neurol Sci. 1996;143(1-2):1-13.

48. Skyler JS, Bergenstal R, Bonow RO, Buse J, Deedwania $\mathrm{P}$, Gale EA, Howard BV, et al. Intensive glycemic control and the prevention of cardiovascular events: implications of the ACCORD, ADVANCE, and VA Diabetes Trials: a position statement of the American Diabetes Association and a Scientific Statement of the American College of Cardiology Foundation and the American Heart Association. J Am Coll Cardiol. 2009;53(3):298-304.

49. Dufouil C, Chalmers J, Coskun O, Besancon V, Bousser MG, Guillon P, MacMahon S, et al. Effects of blood pressure lowering on cerebral white matter hyperintensities in patients with stroke: the PROGRESS (Perindopril Protection Against Recurrent Stroke Study) Magnetic Resonance Imaging Substudy. Circulation. 2005;112(11):1644-1650.

50. Galanth S, Tressieres B, Lannuzel A, Foucan P, Alecu C. Factors influencing prognosis and functional outcome one year after a first-time stroke in a Caribbean population. Arch Phys Med Rehabil. 2014;95(11):2134-2139. 